\title{
Les bassins fluviaux, vecteurs de l'aménagement du territoire
}

\author{
River basins, vehicles for regional development \\ par Christian Bernad \\ Président de l'Association pour l'Aménagement de la Vallée du Lot
}

Rivers (and their tributaries) are living organisms ; their specificity lies in the fact that they are unique in a geographic and time sense with a strong interaction between upstream and dowstream. Whereas, the user only considers the river from his particular point of view. In the past, people rarely took the upstream-dowstream logic into consideration. They used the part of the river that interested them without worrying about the effect of their use on the totality of the waterway. For a long time, uses with direct financial returns had priority over non-commercial uses which were considered without consequence on the local economy.

Water's uniqueness is often confronted with an excess of what are often antagonistic role players : the State and its departments, the local authorities and the users among which are distinguished those who exploit and pollute as well as those who protect. Overexploiting can destroy a river, while overprotecting can undermine the local economy around the catchment Basin.

Rivers are wholly capable of providing leverage for economic development on the condition that an integrated management of the catchment basin is implemented. Consequently, in order to do this, a better understanding of the environment is needed, and the target goals must be defined according to use. This has to be carried out with a permanent concern for maintaining the living system in good condition and within the logic of a lasting development.

\section{I $\square$ LE COURS D'EAU EST UN ORGANISME VIVANT INTERACTIF}

Partons d'un constat. La rivière est un organisme vivant qui a la spécificité d'être unique dans l'espace et dans le temps avec une interdépendance très forte amont-aval, et aval-amont. L'eau qui coule à l'aval vient de l'amont et l'eau de l'amont, si elle n'est pas retenue, va vers l'aval. Rappeler ce schéma scolaire semble puéril, mais force est de constater qu'à travers l'histoire, l'usager n'a trop souvent perçu la rivière que dans l'embrasure de sa fenêtre. Il a utilisé la portion de cours d'eau qui l'intéressait sans se préoccuper si l'usage qu'il en faisait provoquait ailleurs des désordres, mettant parfois en danger de mort ces magnifiques chemins de vie qui ont polarisé, au cours des siècles, les plus prestigieuses cités urbaines et une multitude d'activités économiques.

\subsection{L'aval dépend de l'amont}

Restons dans les évidences : les pollutions de l'amont se retrouvent, quelques heures ou quelques jours plus tard, à l'aval. Mais les pollutions s'additionnent et peuvent perdurer des années, voire des siècles, dans les sédiments. Si l'eau est retenue à l'amont dans des barrages, ou si elle est pompée de manière excessive, il y a pénurie d'eau à l'aval.
Les déboisements à l'amont, la rectification des cours d'eau aggravent les crues et accélèrent la vitesse de l'eau. Quant aux retenues hydroélectriques, elles participent au mouvement en accentuant les variations spontanées du débit.

\subsection{L'amont dépend de l'aval}

Oui quand les extractions de gravier en lit mineur provoquent une érosion régressive des berges, affouillent les piles des ponts, sapent les fondements des digues. Les chaussées, les seuils de toutes sortes, les prélèvements, les pollutions s'opposent à la remontée des poissons migrateurs et, par endroit, à la continuité de la navigation fluviale.

La logique de bassin amont-aval est une notion moderne. Il y a 30 ans, peu de gens se souciaient de ce qui pourrait bien arriver à leur cours d'eau. La rivière était la bonne à tout faire, et à tout évacuer.

\subsection{Le laisser-faire est dangereux}

Les usages à fortes retombées financières directes ont longtemps prévalu (et prévalent encore parfois de nos jours) aux dépens des usages non marchands de type culturel, esthétique ou ludique qui prennent, au demeurant, de plus en plus d'importance sur le plan économique. Les paysages, le patrimoine naturel et bâti, le milieu piscicole, la faune, la 
flore, sont devenus des éléments qui valorisent le " fond de commerce rivière ".

\subsection{Une surabondance d'intervenants}

L'eau est UNE, disions-nous, mais les intervenants sont multiples et parfois antagonistes :

- l'Etat avec ses différents ministères concernés : l'Industrie, l'Environnement, l'Agriculture, le Tourisme, les Finances... et ses nombreux services régionaux et départementaux, l'Agence de l'Eau,

- les Collectivités locales : Régions, Départements, Communes, regroupements de communes, Ententes interdépartementales...

- les usagers où l'on peut distinguer globalement deux groupes : ceux qui produisent, exploitent et quelquefois polluent, et ceux qui protègent (même si ceux qui protègent participent, sans le vouloir, à la pollution générale).

Laisser faire uniquement le premier groupe, ce que l'Etat a favorisé dans le passé, c'est déboucher sur la mort assurée de la rivière par surexploitation.

Laisser faire uniquement le deuxième groupe, c'est aller à l'encontre du développement économique du Bassin, donc accélérer la désertification autour d'une rivière redevenue naturelle. Or, nous le savons, par le passé, les rivières ont trop souffert d'indifférence, c'est, au contraire, en multipliant les activités autour d'elles et en les valorisant que les hommes s'organisent pour les protéger.

\section{II — LA LOI SUR L'EAU DE 1992}

La loi sur l'eau de 1992 a assez bien (pour ne pas dire prudemment) défini les règles d'une gestion intégrée d'un Bassin. La mise en place des Schémas Directeurs d'Aménagement et de Gestion des Eaux ont permis, d'une part d'établir une concertation entre tous les partenaires d'un grand Bassin, et d'autre part de définir un certain nombre d'objectifs, de règles, de recommandations préservant les intérêts de chacun et la sauvegarde des cours d'eau. On attend maintenant la mise en place des SAGE pour planifier la gestion locale, mais, à ce niveau, nous avons quelques inquiétudes car, le moins que l'on puisse dire, c'est que les SAGE ne suscitent pas l'enthousiasme. Cela s'explique. Par définition, le SAGE concerne un espace limité pour accomplir une gestion locale de proximité. L'intention est bonne. Mais, en fait, les SAGE risquent de saucissonner, une fois encore, un sousbassin versant avec des objectifs qui ne seront pas forcément les mêmes d'un SAGE à un autre. Il manque, dans cette loi de 1992, un échelon intermédiaire entre le SDAGE et le SAGE qui pourrait être un Schéma de Cohérence entre les différents SAGE d'un sous-Bassin.

Certes, nous le voyons, l'eau devient chaque jour davantage un enjeu d'appropriation par différents partenaires ayant des visées économiques légitimes ou des préoccupations d'ordre écologique, tout aussi légitimes, en oubliant que la poursuite de ces objectifs représente un coût pour la rivière et pour les collectivités.

\section{III $\square$ LA DIMENSION ÉCONOMIQUE DES COURS D'EAU}

Une rivière représente une dimension économique considérable : pour la production électrique, pour l'irrigation, pour le tourisme. Et dans le même temps, nous assistons à une évasion permanente des richesses économiques issues du cours d'eau. Une part infime de ses richesses retourne à son entretien laissé, le plus souvent, à la charge des Collectivités locales, aidées par les Agences. L'entreprise produit des bénéfices qui ne sont pas, ou si peu, réinvestis pour assurer le maintien et le développement de l'outil. Une telle pratique mènerait tout droit à la faillite de n'importe quelle exploitation. Aussi, serait-il opportun, avant d'engager tout projet d'envergure, de dresser systématiquement un bilan économique et d'évaluer son impact sur les autres usages.

Nous assistons cependant à des initiatives réconfortantes dans ce domaine, notamment au niveau des Agences de l'Eau qui considèrent de plus en plus la rivière et son Bassin versant comme un support de l'Aménagement du Territoire.

La mise en place, par exemple, d'une Politique Territoriale contractuelle à l'Agence Adour-Garonne est une avancée significative. La multiplication, en France, d'Institutions Interdépartementales de collectivités locales démontre également l'importance économique croissante des cours d'eau.

L'eau des rivières permet, en effet :

- de garantir l'eau potable nécessaire aux collectivités humaines et aux productions animales en quantité et en qualité, - d'assurer la production végétale par l'irrigation, et de promouvoir l'implantation d'unités agro-alimentaires,

- d'assurer le transfert des eaux usées, et de participer à une dépollution par autoépuration,

- d'être une voie de transport,

- de favoriser certaines installations industrielles,

- de produire l'électricité hydraulique dont la souplesse d'utilisation est irremplaçable, et de participer d'une certaine manière au soutien des débits d'étiages,

- d'être un milieu de vie : pour la faune et la flore aquatique, de constituer des zones humides indispensables,

- de favoriser, de plus en plus, les activités nautiques, par exemple :

- le tourisme fluvial, actuellement en pleine expansion, et qui se révèle être un produit de choix pour l'étalement des vacances ; le tourisme fluvial se pratique également sur les grandes retenues de l'amont ; là où il se développe, il induit des équipements considérables,

- la pratique du canoë-kayak, de l'aviron, de la voile...

- de pratiquer la baignade et la pêche. N'oublions jamais que demain, seules les rivières où l'on pourra pêcher et se baigner, seront fréquentées par les touristes,

- de développer l'écotourisme, phénomène relativement récent qui privilégie l'observation et l'éducation.

Enfin, l'eau des rivières doit permettre aux hommes de se ressourcer, de rêver, de se rééquilibrer face à la démesure de la vie urbaine et industrielle. C'est sans nul doute sa fonction d'aménagement du territoire la plus importante. L'eau est un bien inaliénable de l'humanité.

\section{IV — VERS UNE GESTION INTÉGRÉE}

Il est de notre devoir, pour le présent et le futur, de rééquilibrer les usages de l'eau, en favorisant, en particulier, les intérêts de ceux qui s'identifient et vivent en riverains citoyens.

Les rapports de force existants n'évolueront que par la concertation autour d'un projet de gestion intégrée. 
L'aménagement du territoire, comme son nom l'indique, ne doit pas être une somme de grands équipements qui produisent isolément des bénéfices, sans tenir compte de la logique d'ensemble du Bassin, mais au contraire l'aménagement du territoire doit assurer le meilleur équilibre possible des usages de l'eau et des rivières, en tenant compte de toutes les valeurs et surtout en maintenant la bonne santé et la vie de la rivière.

Les conditions nécessaires pour réaliser une gestion intégrée génératrice de richesses et d'emploi, pourraient s'établir selon quelques grands principes :

- d'abord choisir le cadre de l'action, l'envergure d'un sousbassin versant comparable à ceux du Lot, de la Dordogne, de la Vilaine, du Cher .... nous semblant le mieux adapté,
- ensuite se libérer des frontières administratives en créant un organisme interdépartemental ou interrégional, ou intercommunal.... suivant la volonté locale et où se retrouveront les utilisateurs de la rivière,

- approfondir la connaissance du milieu,

- définir les objectifs à atteindre par usage, en concertation avec les élus et les usagers, tout en respectant le SDAGE,

- enfin, informer, pour ne pas dire éduquer, les populations riveraines en commençant par l'Ecole.

“ Le grand défi du XXIe siècle sera de concilier, pour le plus grand profit des générations futures, les exigences économiques et les impératifs environnementaux. Ce défi, c'est le développement durable de nos rivières". 